\title{
Interneuron Progenitors Attenuate the Power of Acute Focal Ictal Discharges
}

\author{
Estanislao De la Cruz • Mingrui Zhao $\cdot$ Lihua Guo • \\ Hongtao Ma $\cdot$ Stewart A. Anderson • \\ Theodore H. Schwartz
}

Published online: 12 July 2011

(C) The American Society for Experimental NeuroTherapeutics, Inc. 2011

\begin{abstract}
Interneuron progenitors from the embryonic medial ganglionic eminence (MGE) can migrate, differentiate, and enhance local inhibition after transplantation into the postnatal cortex. Whether grafted MGE cells can reduce ictal activity in adult neocortex is unknown. We transplanted live MGE or killed cells (control) from pan green fluorescent protein expressing mice into adult mouse sensorimotor cortex. One week, 2 and $1 / 2$ weeks, or 6 to 8 weeks after transplant, acute focal ictal epileptiform discharges were induced by injection of 4-aminopyridine (4-AP) $2 \mathrm{~mm}$ away from the site of transplantation. The local field potential of the events was recorded with 2 electrodes, 1 located in the 4-AP focus and the other 1 in the transplantation site. In all control groups and in the 1-week live cell transplant, 4-AP ictal discharges revealed no attenuation in power and duration from the onset site to the site of transplantation. However, 2.5 or $6 \sim 8$ weeks after MGE
\end{abstract}

Estanislao De la Cruz and Mingrui Zhao contributed equally to this study

Electronic supplementary material The online version of this article (doi:10.1007/s13311-011-0058-9) contains supplementary material, which is available to authorized users.

E. De la Cruz $\cdot$ M. Zhao $\cdot$ H. Ma

Departments of Neurological Surgery, Weill Cornell Medical

College, New York Presbyterian Hospital,

New York, NY 10065, USA

T. H. Schwartz $(\bowtie)$

Departments of Neurological Surgery, Neurology and Neuroscience,

Weill Cornell Medical College, New York Presbyterian Hospital,

New York, NY 10065, USA

e-mail: schwarh@med.cornell.edu

L. Guo $\cdot$ S. A. Anderson $(\square)$

Department of Psychiatry, Weill Cornell Medical College,

New York Presbyterian Hospital,

New York, NY 10065, USA

e-mail: saa2007@med.cornell.edu transplants, there was a dramatic decrease in local field potential power at the MGE transplanted site with little decrease in ictal duration. Surprisingly, there was no relationship between grafted cell distribution or density and the degree of attenuation. As remarkably low graft densities still significantly reduced discharge power, these data provide further support for the therapeutic potential of interneuron precursor transplants in the treatment of neocortical epilepsy.

Keywords Cerebral cortex · Epilepsy · Seizure $\cdot$ Interneuron progenitor $\cdot$ Transplantation

\section{Introduction}

It is estimated that more than $30 \%$ of all epilepsy patients are refractory to conventional anti-epileptic medication [1]. A proportion of these patients may be candidates for surgery to remove the epileptic focus $[1,2]$, but a significant fraction continue to have seizures without hope of further treatment.

The loss of neuronal inhibition has been demonstrated in a variety of epilepsy models and proposed as a possible mechanism for increased excitability. In fact, several interneuron subgroups have been associated with the suppression of seizures, including chandelier cells expressing parvalbumin (PV) [3] and basket cells expressing either somatostatin (SST), and/or neuropeptide $\mathrm{Y}$ [4]. As a result, transplantation of inhibitory interneurons into an epileptic focus may be a potential therapy for cases of pharmacoresistant epilepsy [5].

A variety of transplantation strategies have been used to suppress epileptic activity, such as the use of immortal cell lines engineered to produce gamma-Aminobutyric acid (GABA) [6], and embryonic stem cell-derived adipose 
tissue engineered to release the potent anti-epileptic adenosine $[7,8]$. However, only recently have advances in understanding cortical interneuron origins [9] and appreciation of their remarkable ability to migrate and survive after transplantation into neonatal or adult cortex [10] ultimately led to interneuron transplantation studies to treat seizures in rodents [11-13]. Although species differences with primates appear to exist, in rodents and ferrets most cortical interneurons, including the PV and the SST expressing subgroups, originate in the medial ganglionic eminence (MGE) of the subcortical telencephalon [14]. Genetic fatemapping and transplantation studies indicate that within the MGE there is a bias for PV or SST expressing interneurons to originate from ventral or dorsal MGE regions, respectively [15-17]. Transplanted MGE cells not only differentiate into mature GABAergic (gamma-aminobutyric-acid-releasing) interneurons, but also form inhibitory synapses that increase GABAergic synaptic transmission onto adjacent pyramidal cells [18] and alter cortical pyramidal neuron plasticity [19].

Three recent studies have indeed presented evidence that transplantation of MGE-derived interneuron precursors can reduce seizure activity. In the first study, transplants into neonatal neocortex reduced generalized seizures and modestly improved survival in a genetic model of cortical hyperexcitability [12]. In the second study, reduced seizure threshold produced in adult mice by selective killing of hippocampal interneurons was normalized by transplantation of MGE-derived interneurons [13]. Finally, MGEderived neural stem cells transplanted into adult hippocampus reduced kainic acid-induced kindled seizures, although in this study the grafts included many astrocytes [11]. Despite these advances, important questions remain about this promising therapy. For example, how soon after transplantation do the anti-seizure effects appear? Is this therapy use effective against other types of ictal events, such as focal neocortical seizures? Finally, is there a rapid method to screen transplant efficacy in an acute in vivo model?

To address these questions, we investigated the relationship between the quantity of surviving MGE transplants and their anti-epileptic effect using a model of focal injection of 4-aminopyridine (4-AP), which is a potent convulsant that acts by blocking slowly inactivating potassium currents [20] and enhancing the release of synaptic neurotransmitters [21], which elicits focal ictallike events that initiate spontaneously and propagate horizontally [22]. Although the pharmacoresistance of focal neocortical 4-AP injection in vivo has not been tested, intraperitoneal injection and in vitro data indicate at least partial pharmacoresistance [23-27]. Although the 4-AP model is not a model of chronic epilepsy, it is advantageous as a rapid throughput screen of anti-epilectic therapy [28]. Herein, we describe that MGE transplants dramatically reduce seizure propagation indicating that acute focal injection of 4-AP can be used as a high throughput model to assess cell transplant therapeutic efficacy.

\section{Materials and Methods}

\section{In Vivo Transplantation}

All experimental procedures were approved by the Weill Cornell Medical College Animal Care and Use Committee following the National Institutes of Health guidelines. Green fluorescent protein (GFP) expressing transgenic mice were maintained on a CD1 background. At 13 days of gestation (E13.5), dams were sacrificed and the GFP+ embryos were placed in ice-cold Hank's buffer. The brains were removed and the ventral forebrain was exposed by removing the dorsal and lateral region of cortex. MGE cells are then harvested from 250- $\mu \mathrm{m}$ sections by first gently freeing the dorsal and ventral MGE from the adjacent tissue and then by mechanically dissociation (Fig. 1). Cells were then re-suspended in Neurobasal/B27 medium (Gibco/Invitrogen Grand Island, $\mathrm{NY}$ ) and kept on ice until transplantation.

Adult CD1 mice 6 to 8 weeks old were initially anesthetized with 1.5 to $2 \%$ isoflurane in $70 \% \mathrm{~N}_{2}: 30 \% \mathrm{O}_{2}$ by face mask and mounted in a stereotaxic frame. Mice were maintained on $1.5 \%$ isoflurane in $70 \% \mathrm{~N}_{2}: 30 \% \mathrm{O}_{2}$. An incision was made in the midline to expose the skull. A burr hole was drilled above the motor cortex. A total of $1.5 \times 10^{4}$ cells per mouse in $1.5 \sim 2.0 \mu \mathrm{l}$ volume was stereotaxically injected into the motor cortex (coordinates related Bregma: $-0.7 \mathrm{~mm}$ anterior, $-1.0 \mathrm{~mm}$ lateral, and $-1.0 \mathrm{~mm}$ ventral) by a Nanoject II injector (Drummond Scientific Broomall, PA). Dead cells transplanted by the same procedure into separate groups of animals were used as controls. Dead cells were generated by taking an aliquot of the same GFP+MGE cells prepared for live cell transplants, but killing them by freezing and thawing (at $-80^{\circ} \mathrm{C}, 1$ minute $\times 3$ ) immediately prior to transplantation. No GFP-expressing cells were detected in sections through the region of dead-cell transplants (data not shown).

\section{Epileptogenesis and Electrophysiology}

In 3 separate experiments, either 1, 2.5, or $6 \sim 8$ weeks after cell transplantation, adult male CD1 mice (27 to $35 \mathrm{~g}$ ) were initially anesthetized with 1.5 to $2 \%$ isoflurane and maintained on $1.0 \sim 1.5 \%$ isoflurane in $70 \% \mathrm{~N}_{2}: 30 \% \mathrm{O}_{2}$ by face mask. The mouse was maintained to be stable and carefully monitored as previously described [22]. Animals were placed in a stereotaxic frame. An $3 \times 4-\mathrm{mm}$ cranial window was opened over 1 hemisphere to expose somato- 
Fig. 1 Transplantation of dorsal medial ganglionic eminence (dMGE) or ventral medial ganglionic eminence (vMGE) cells into the adult cortex results in longterm survival, migration, and differentiation of interneurons. (a) The medial ganglionic eminence (MGE) of an E13.5 embryo is dissected into dMGE and vMGE domains. (b) Immunofluorescence labeling for GFP in a $12-\mu \mathrm{m}$ coronal section showing the location of an injection site 1 day after transplantation into the adult cortex (GFP: green; Ki67: red; and DAPI: blue). Co-labeling of GFP and the cell cycle marker $\mathrm{Ki}-67$ is very rare $(1 \%)$. Scale bar: $200 \mu \mathrm{m}$. (c) Distributions of MGE cells 6 to 8 weeks after transplantation. The left panel shows the distribution of surviving MGE cells (green) in the adult neocortex. The right panel shows the migration pattern of both dMGE ( $n=7$ mice) and vMGE cells ( $n=9$ mice). The X-axis show rostral-caudal distance from the injection site. The Y-axis shows the percentage of cells in each $250-\mu \mathrm{m}$ bin. $\mathrm{R}=$ rostral; $\mathrm{C}=$ caudal. (d) Distributions of MGE cells 2.5 weeks after transplantation. The left panel shows the distribution of transplanted MGE cells (green) in the adult neocortex. Box insert shows higher magnification of the cells. The right panel shows the migration pattern of the MGE cells ( $n=6$ mice). (e) Distributions of MGE cells 1 week after transplantation. The left panel shows the distribution of surviving MGE cells (green) in the adult neocortex. The box insert shows higher magnification of the cells. The right panel shows the migration pattern of the MGE cells $(n=6$ mice) a

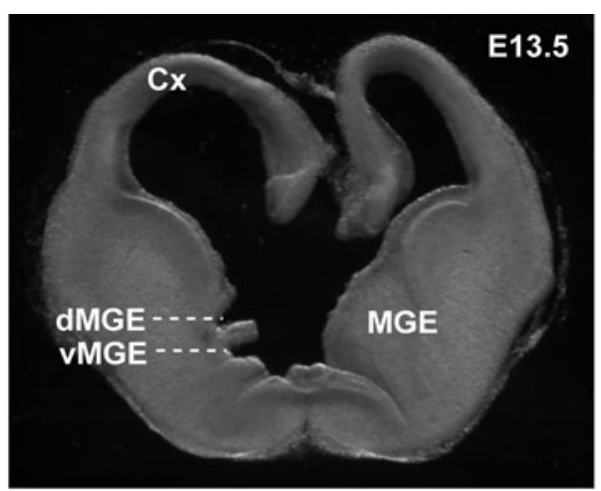

c
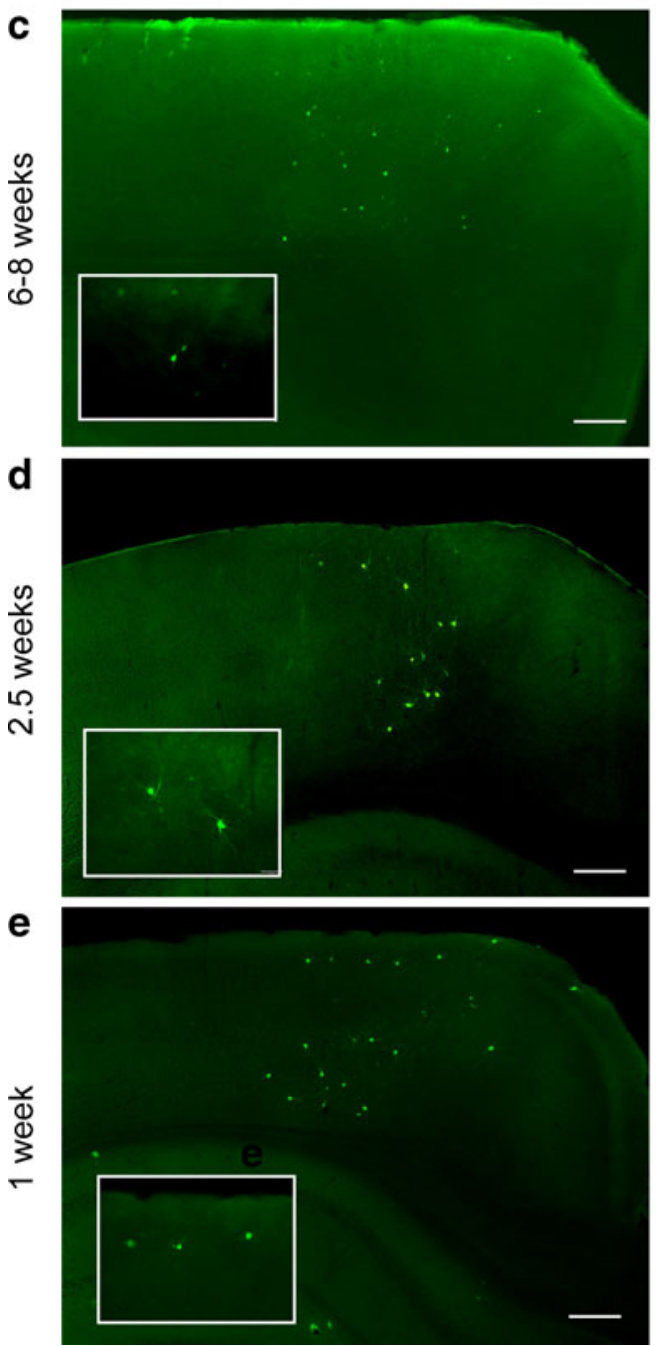
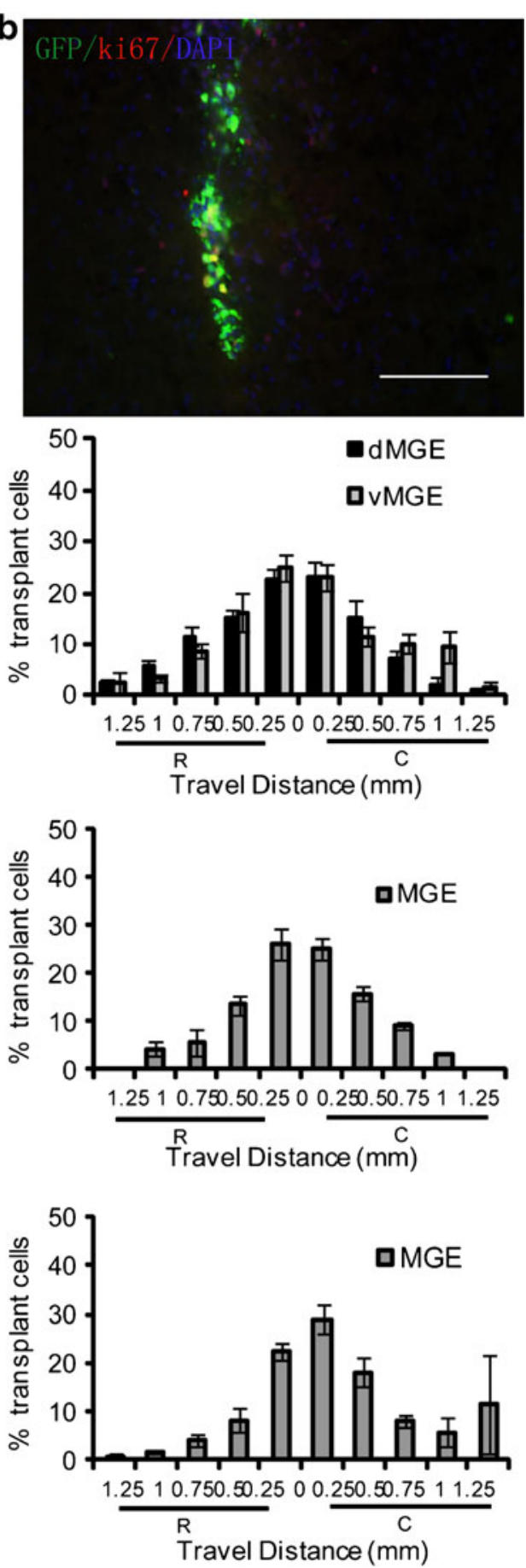

sensory cortex. Ictal discharges were induced by injecting 4-AP (Sigma, St. Louis, MO $15 \mathrm{mM}$ in $0.9 \%$ saline, $0.5 \mu \mathrm{l}$ ) through a single-barreled glass microelectrode (impedance, 2-4 M $\Omega$ ) using a Nanoject II Injector placed $2 \mathrm{~mm}$ away from the site of the transplant. The local field potential (LFP) was recorded with 2 electrodes. Ictal onset was recorded from the same electrode used for 4-AP injection of the local field potential (LFP-1). Ictal propagation was recorded from the site of transplantation of the local field potential (LFP-2), which was identified by the injection burr hole. The LFP-2 electrode was filled with $0.9 \%$ saline (impedance, 2-4 M $\Omega$ ). LFP was amplified (gain $=1000$ ) and filtered between 0.1 and $1000 \mathrm{~Hz}$ using an AC/DC Differential Amplifier (Model 3000, A-M Systems, Carlsborg, WA), and recorded by a CED Power 1401/Spike2 system at $1000 \mathrm{~Hz}$ (Cambridge Electronic Design, Cambridge, UK). The amplitude and duration of spontaneous ictal epileptiform events were recorded at each of the two LFP electrodes, 1 in the 4-AP focus (LFP-1) and the other in the region of the cell transplants (LFP-2) to determine propagation efficacy. 
Immunohistological Staining

After the recordings were complete, animals were transcardially perfused with $4 \%$ paraformaldehyde in phosphate buffered saline and postfixed overnight at $4{ }^{\circ} \mathrm{C}$. Brains were then sectioned in the coronal plane at $50 \mu \mathrm{m}$ on a vibratome. Primary antibodies used for immunofluorescence labeling included anti-rabbit GFP (Molecular Probes, Eugene, OR 1:2000), anti-mouse parvalbumin (Swant, Bellinzona, Switzerland 1:5000), anti-rat somatostatin (Chemicon, Temecula, CA 1:200), anti rabbit Ki-67 antibody (Thermo Scientific, Rockford, IL 1:500), and anti-rabbit Iba1 (ionizing calcium-binding adaptor molecular 1) antibody (Wako Chemicals, Osaka, Japan 1:1000). The following secondary antibodies were used: anti-rabbit 488 (Molecular Probes, 1:500), anti-mouse 568 (Molecular Probes, 1:500), and anti-rat Cy5 (Jackson ImmunoResearch Laboratories, Inc, West Grove, PA 1:500). The cell distribution was calculated by taking the average of the sections $200 \mu \mathrm{m}$ apart, which constitutes a bin, and multiplying the result by 5 ("see Supplemental Material for details").

\section{Data Analysis}

The offline analysis was performed using custom analysis software written in MATLAB (MathWorks Natick, MA). Ictal onset could be easily determined by visual analysis of the LFP-1 recording based on the morphology of the seizures. In the 4-AP model, most epileptiform discharge start with a large spike-and-wave, followed by a recruiting rhythm that evolves into repetitive spike-and-wave discharges that dissipate with progressive decrease in amplitude and increase in interspike interval, epileptiform events last for an average of 54.6 \pm 4.4 seconds. To eliminate any ambiguity in defining seizure onset, seizures that initiate with a large spike were included ("see Supplemental Material Fig. S1 for details"). These discharges bear a striking resemblance to spontaneous human seizures [29]. Ictal termination was determined by eye by 2 separate nonblinded observers independently assessing when the tracings returned to their baseline, pre-ictal activity. Total LFP ( $\Sigma$ LFP) power was calculated as an integral of the area above 2 standard deviation baseline activity under the response curve of each event. Baseline activity was measured for a 2-second epoch prior to the initiating spike. The ratios of $\Sigma$ LFP power and duration from LFP-1 were compared to LFP-2 to establish the effect of MGE cells transplantation on each epileptiform event. Comparisons between the 2 groups were performed with 2-tailed $t$ tests. Significance was set at $p<0.05$. For all figures, error bars represent standard error of the mean.

\section{Results}

Survival and Differentiation of MGE-Derived Cells Transplanted into Adult Neocortex

MGE-derived progenitors transplanted into both neonatal and adult mouse cortex have been shown to migrate, survive, and differentiate into several subgroups of cortical interneurons $[10,15,17,18]$. Relative to progenitors of the cortex, striatum, or hypothalamus, this migration was found to be unique to MGE-derived progenitors [10]. Accordingly, in our experiments, 1 day after transplantation into layers 2 to 6 of the 6- 8-week-old cortex, GFP +cells were distributed close to the injection site (Fig. 1b) with many cells exhibiting a bipolar morphology typical of migrating interneurons (Fig. 1c). Consistent with previous findings that MGE cells exit the cell cycle at the time of transplantation or shortly after transplantation [30], there was minimal co-labeling of GFP and Ki-67 at 1 day posttransplant (Fig. 1b). Approximately $16 \mathrm{~h}$ after cell transplantation, the proliferation of MGE cells was examined by Ki-67 immunofluorescence labeling. Ki-67 is a nuclear protein, expressed during late G1 (Gap 1), S (Synthesis), M (Mitosis), and G2 (Gap 2) phases. Less than $1 \%$ of the GFP + cells labeled for Ki-67 (Fig. 1b), suggesting that only a small percentage of the transplanted embryonic day (E) 13.5 MGE cells were still undergoing cell division in the adult brain after $24 \mathrm{~h}$. Indeed, no tumors or cysts were found in any of the transplants analyzed in this study.

Two months after transplantation, extensive migration of cells had occurred to a distance of approximately greater than $1 \mathrm{~mm}$ away from the injection site in all directions (Fig. 1c). There was no significant difference in the migration of cells arising from the dorsal MGE (dMGE) compared with the ventral MGE (vMGE) (Fig. 1c). The transplanted cells tended to have multipolar dendritic morphologies, and were aspiny or sparsely spiny. Co-labeling with GABA revealed that $54.01 \pm 4.39 \%$ of dMGE transplants and $57.44 \pm 3.56 \%$ of vMGE transplants express detectable levels of GABA (Fig. 2a, d). Of note, GABA-labeling in these preparations is weak because glutaraldehyde fixation was not used due to its weakening of labeling for the other markers. Although a relatively small population of multipolar, sparsely spiny, cholinergic interneurons of the striatum may also originate in the MGE, we have detected no co-labeling for the cholinergic marker ChAT in MGE transplants into either the neonatal or adult neocortex (data not shown).

To determine whether MGE-derived progenitors acquire the predicted neurochemical characteristics of cortical interneurons after transplantation into adult neocortex, transplanted GFP+ cells were examined by immunofluorescence for the interneuron subgroup markers PV (Fig. 2c) or SST (Fig. 2b). Counts of cells in triple-labeled (PV-SST-GFP) sections revealed that in 
A

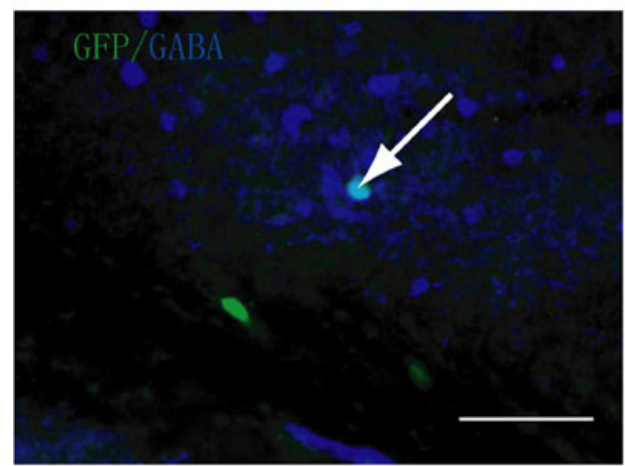

C

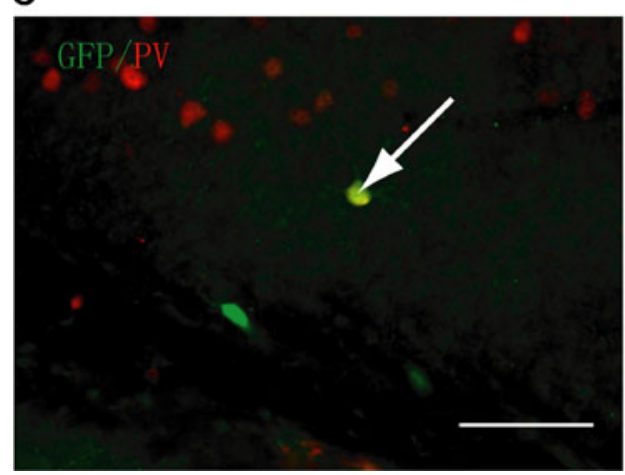

B
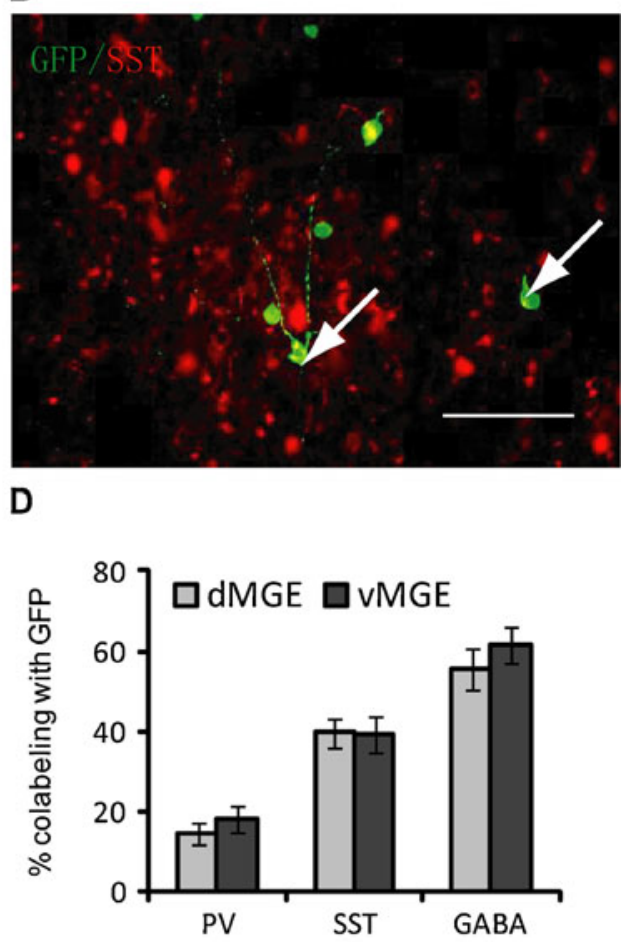

Fig. 2 Transplantation of dorsal medial ganglionic eminence (dMGE) or ventral medial ganglionic eminence (vMGE) cells into the adult cortex results in differentiation into the interneurons. (a) Immunolabeling of $50-\mu \mathrm{m}$ coronal sections taken 60 days post- transplant. The majority of these cells express detectable levels of gamma-Aminobutyric acid (GABA). Arrow shows co-labeling with green fluorescent protein (GFP). Scale bar: $100 \mu \mathrm{m}$. (b) Confocal images showing co-labeling for GFP and somatostatin (SST) from a dMGE transplant (arrows). Scale

dMGE transplants $(n=7), 39.54 \pm 3.80 \%$ express SST and $12.49 \pm 2.73 \%$ express PV. In vMGE transplants $(n=9)$, $38.29 \pm 4.86 \%$ express SST and $16.59 \pm 3.89 \%$ express PV (Fig. 2b). In summary, we did not see a difference in expression of SST and PV from either dMGE or vMGE. At 1 week, 2.5 weeks, and 6 8 weeks after transplantation, $>80 \%$ of the cells transplanted were located within $500 \mu \mathrm{m}$ of the injection site in both rostral and caudal directions.

\section{Impact of MGE Transplants on Seizure Propagation}

We assessed the ability of MGE-derived transplants to influence epileptiform activity using focal injection of 4-AP, a potent convulsant and $\mathrm{K}+-$ channel blocker [20]. In 6 to 8 weeks after transplantation, a focus was created with 4AP located $2 \mathrm{~mm}$ away from the transplant site. LFPs were recorded from the 4-AP focus (LFP-1) and the cell transplant site (LFP-2), respectively (Fig. 3a). Previous studies have found that neural transplants can transiently decrease seizure threshold or create a focus in a subset of cases [31, 32]. For this reason, we recorded a 15-minute baseline period from both LFPs prior to injecting the 4-AP. bar: $100 \mu \mathrm{m}$. (c) Confocal image showing co-labeling of GFP cells with parvalbumin (PV) from a vMGE transplant. Arrow shows co-labeling with GFP. Scale bar: $100 \mu \mathrm{m}$. (d) Bar graph showing the percentage of $\mathrm{PV}$, SST, and GABA co-labeling with GFP in dMGE and vMGE transplants. Because a subpopulation of vMGE cells migrate through the dMGE proliferative zone, some of the $\mathrm{PV}+$ cells in the dMGE transplants in all probability originated in the vMGE (see Wonders et al., 2008 [15]). dMGE, $n=8$ mice; $v M G E=9$ mice

No abnormal activity, such as interictal spikes, polyspikes, or ictal discharges were identified in any of the experiments (data no shown). This suggests that neither the injected control cells nor MGE cells created epileptiform activity.

In the control groups, the ratio of seizure duration and seizure $\Sigma$ LFP power between killed cell site and 4-AP site were $0.994 \pm 0.004$ and $1.018 \pm 0.152$ ( $n=6$ mice; 81 events). This indicates that each ictal event quickly propagates from the focus to the cell transplant site $2 \mathrm{~mm}$ away (Fig. 3b). However, the ratio of seizure $\Sigma$ LFP power decreased to $0.330 \pm 0.127$ ( $n=8$ mice; 143 seizures) and $0.329 \pm 0.082$ ( $n=9 ; 169$ events) in the dMGE and vMGE groups, respectively. Both dorsal and ventral MGE transplants significantly reduce the power of 4-AP induced discharges relative to killed cell controls (dMGE vs control; $p<0.001$; vMGE vs control; $p<0.01$; Fig. $3 \mathrm{c}$ ). There was no significant difference between dMGE group and vMGE group in the ratio of ictal $\Sigma$ LFP power $(p>0.99)$. The ratio of ictal duration of dMGE group and vMGE group changed to $0.960 \pm 0.016$ and $0.920 \pm 0.032$, respectively. Only vMGE cells significantly reduced seizure duration ( $p<0.05$; Fig. $3 c)$. Analysis of each individual event also showed that MGE 

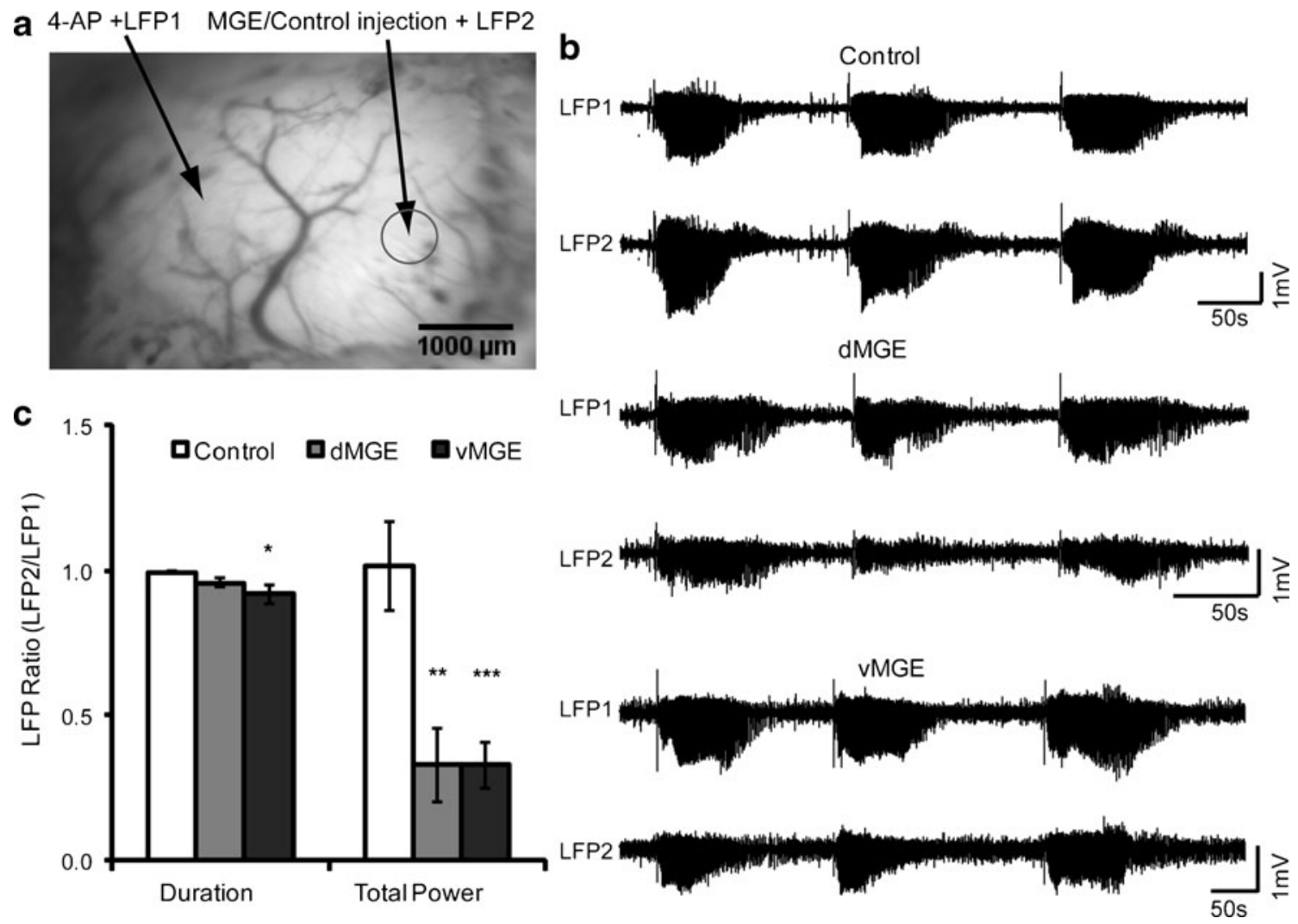

Fig. 3 Medial ganglionic eminence (MGE) transplants attenuate propagation of 4-aminopyridine (4-AP) seizures. (a) Image of the cortical surface demonstrating the locations of the local field potential (LFP) with ictal onset recorded from the same electrode used for 4-AP injection of the local field potential (LFP-1) electrode and the 4-AP focus (left), and the ictal propagation was recorded from the site of transplantation of the local field potential (LFP-2) electrode on the MGE/control cells injection site (right) Scale bar: $1000 \mu \mathrm{m}$. (b) Examples of 4-AP seizures recorded at LFP-1 and LFP-2 during control (top), dorsal MGE (dMGE) transplant (middle) and ventral

MGE (vMGE) transplant (bottom) experiments. Note that LFP-1 and LFP-2 are nearly identical for the control experiments. In contrast, both the dMGE and vMGE transplants cause a clear reduction in seizure amplitude. (c) Effect of transplantation on ictal duration and power in control ( $n=6$ mice, 81 seizures), dMGE ( $n=7$ mice, 143 seizures), and vMGE ( $n=9$ mice, 169 seizures) experiments. Quantified normalized results showed both dMGE and vMGE transplants significantly reduced the total seizure power and vMGE transplants reduced the seizure duration. $\left({ }^{*} p<0.05 ;{ }^{*} p<0.01 ; * * * p<0.005\right)$

cells reduced seizure propagation ("see Supplemental Material for details").

\section{Effect of Transplant is Independent of Cell Number}

For the 6- 8-week transplant groups, the number of transplanted cells was estimated by counting the number of cells in 4 bins across a 1-mm distance. The average cell number was $264 \pm 69$ ( $n=7$ mice) and $224 \pm 59$ ( $n=9$ mice) for dMGE and vMGE groups, respectively (not significant; Fig. 4a). To further investigate the relationship between the number of transplant cells and the attenuation of ictal power propagation, we plotted cell transplant number against the ratio of LFP-2/LFP-1 power, a value that represents efficacy in attenuation of propagation. We presumed that inhibitory neuronal cell density would correlate with efficacy, consistent with the notion that the transplanted cells were making inhibitory synapses on the surrounding pyramidal cells and dampening down the excitatory activity, as has been

hypothesized in prior publications [12]. Surprisingly, there was no significant correlation between cell number and the LFP-2/LFP-1 ratio (Fig. 4b). These results indicate that the mechanism of action of MGE transplants to attenuate ictal power is independent of transplant cell number, at least above the level of a surprisingly low threshold (Fig. 4b).

\section{Latency of Transplantation Effect}

Since the reduction in ictal power did not correlate with cell transplant number, suggesting that inhibitory synaptogenesis may not play a direct role in the effect of interneuron precursor transplantation, we explored the possibility that an effect might be recognized earlier than $6 \sim 8$ weeks after transplantation, before the majority of synapses are formed. For this approach, we repeated our transplantation experiments and recorded the effect at an earlier time point, 2.5 weeks posttransplantation (Fig. 5a). The ratio of ictal duration between LFP-2 and LFP-1 was $1.013 \pm 0.17$ ( $n=4$ mice; 53 events) in 

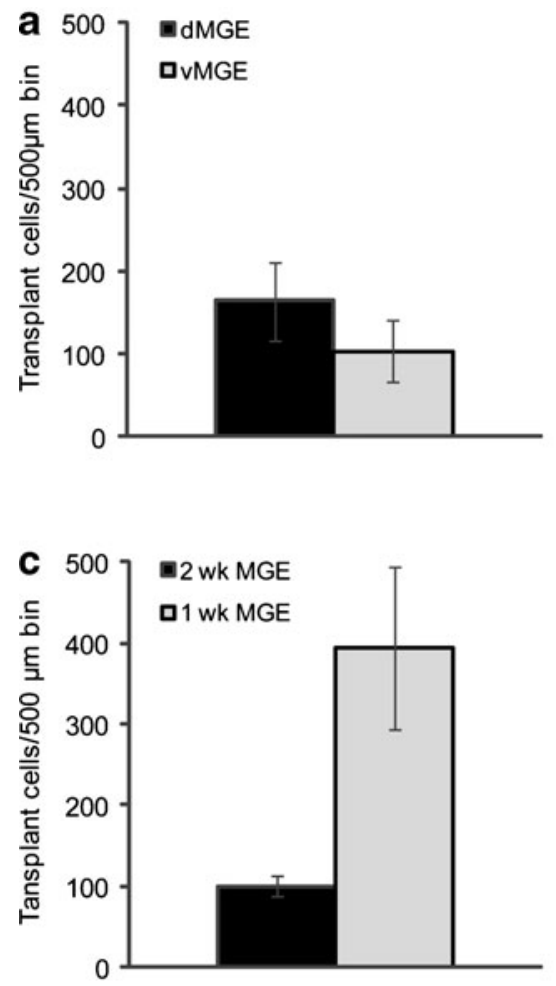

Fig. 4 Transplant density does not correlate with efficacy at reducing ictal power. (a) Both dorsal medial ganglionic eminence (dMGE) and ventral medial ganglionic eminence (vMGE) have equivalent but low cell density. (b) The ratio of total ictal power between the 4aminopyridine (4-AP) focus and the transplantation site local field potential (LFP) with ictal onset recorded from the same electrode used for 4-AP injection of the local field potential (LFP-1) and ictal propagation was recorded from the site of transplantation of the local field of potential (LFP-2), which is independent of survival number. The linear regression lines are as follows: dMGE, $y=-141.59 \mathrm{x}+$

the control group, respectively and $0.984 \pm 0.010(n=6$ mice, 59 events) in the MGE transplant group, which was not significantly different (Fig. 5b). When we compared the ratio of ictal power in LFP-2 and LFP-1 there was a dramatic decrease, from $1.152 \pm 0.017$ in the control group to $0.435 \pm$ 0.099 in the MGE group ( $p=0.039$; Fig. $5 b$ ). As in the 6 - to 8 -week transplants, there was no effect of cell number of efficacy and a surprisingly small number of cells was capable of exerting a significant effect (Fig. 4c, d).

To further investigate the effect of early transplantation, we studied ictal power 1-week post-transplant. At this early time point, there was no effect of the transplants on either duration or power (Fig. 5 d-f).

\section{Discussion}

It has been more than 100 years since Thompson's earliest attempts at neural grafting in the brain [33], yet only recently
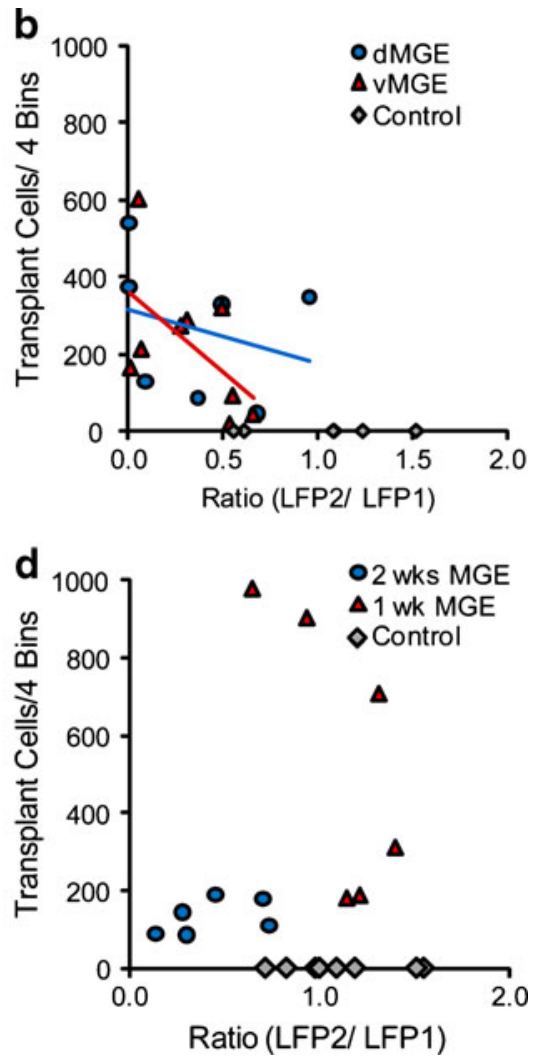

317.17. $\mathrm{R}^{2}=0.0815, n=7$ mice; vMGE, $\mathrm{y}=-417.01 \mathrm{x}+361.44 . \mathrm{R}^{2}=$ $0.3338, n=9$ mice; and control, $n=6$ mice. (c) Average cell density at 1-week post-transplant shows a higher number of cells than 2.5 and 6 to 8 weeks. (d) The ratio of total ictal power between the 4-AP focus and the transplantation site is independent of cell number at these earlier time points, as well. The linear regression lines are -2.5 weeks, $y=$ 84.494 $\mathrm{x}+95.61 . \mathrm{R}^{2}=0.2039, n=6$ mice; and 1 week, $\mathrm{y}=-905.11 \mathrm{x}+$ 1552.9. $\mathrm{R}^{2}=0.481, n=6$ mice; and controls, $n=8$ mice. MGE $=$ medial ganglionic eminence

have researchers begun the modern era of neural transplantation. The strategy of using stem cells as a drug delivery system for brain tumors has been applied in the rodent for $>10$ years [34]. Additional studies showed that the delivery of glial cell line-derived neurotrophic factor through engineered human neural progenitor cells improved neurons survival and function in the rodent models of Huntington's disease, Parkinson's disease, and amyotrophic lateral sclerosis [35-38]. Likewise, transplantation of MGE cells into the epileptic cortex has been proposed as a cell-based therapy for medically intractable epilepsy. Early approaches involved transplants of immortalized cortical neurons that reversibly overexpress GABA [6, 39]. Transplants of these cells into the substantia nigra or dentate gyrus suppressed kindled hippocampal seizures [40]. Another promising approach involved the transplantation of embryonic stem cells engineered to produce adenosine $[7,8]$. The current study takes an alternate approach from these studies in that the cells used for transplantation derive from the embryonic source of most 

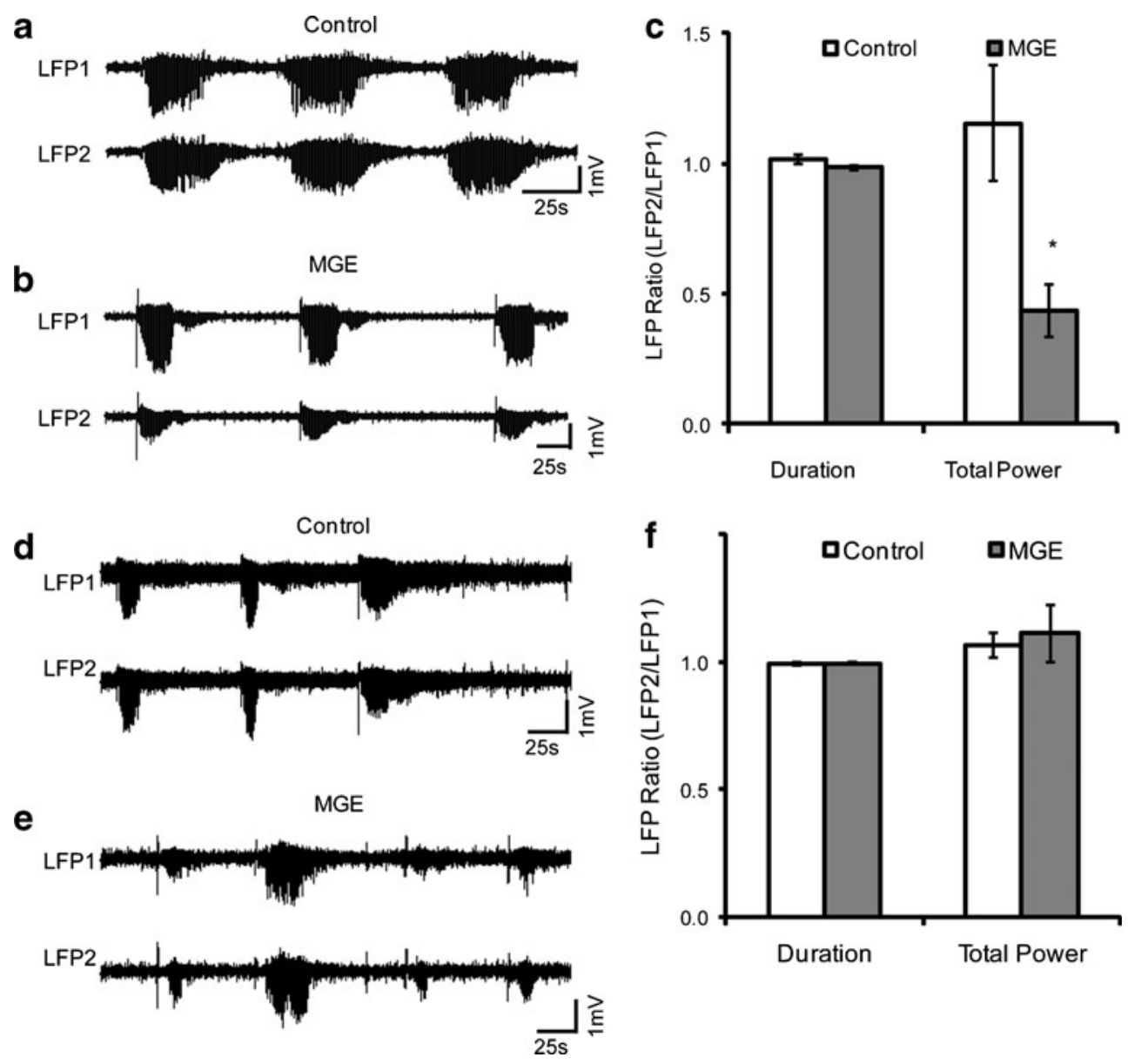

Fig. 5 Early effect of interneuron transplant on seizure propagation. (a) The local field potential (LFP) recording at the 4-aminopyridine (4-AP) injection site, where seizures initiate (top trace), and at the location of a killed-medial ganglionic eminence (MGE) cell control transplant 2.5 weeks after transplantation shows no attenuation in power (bottom trace). (b) As early as 2.5 weeks after transplantation of MGE cells, a clear reduction in seizure power is identified at the site of transplantation, compared with the site of initiation. (c) Comparison of seizure duration and power in control ( $n=4$ mice, 53 seizures) and transplant ( $n=6$ mice, 59 seizures) groups shows

cortical GABAergic interneurons, namely the MGE of the subcortical telencephalon. Prior reports have demonstrated the efficacy of this approach in early postnatal rodent neocortex and adult hippocampus $[12,13,18]$. In this report, we confirm the therapeutic efficacy of these transplants and extend the indications to adult rodent neocortex. Also, in particular, an acute model of neocortical seizures can be used as a rapid throughput screen of transplant therapy. Although we have shown the efficacy of MGE transplants in this model, it is unclear how MGE transplants compare with more traditional anticonvulsant therapy, such as standard anti-epileptic medications, because a direct comparison was not performed.

Alvarez-Dolado et al. [18] have shown that grafted MGE cells migrate up to $5 \mathrm{~mm}$ in the postnatal rodent brain and demonstrate firing properties of mature inter- significant early reduction in power. ${ }^{*} p<0.05$. (d) LFP recording at the 4-AP injection site, where seizures initiate (top trace), and at the location of a killed-MGE cell control transplant 1 week after transplantation shows no attenuation in power (bottom trace). (e) No clear reduction in seizure power is identified at the site of transplantation, compared with the site of initiation. (f) Comparison of seizure duration and power in control ( $n=5$ mice, 36 seizures), and transplant ( $n=6$ mice, 56 seizures) groups shows no significant effect 1 week after transplant

neurons. Likewise, adjacent pyramidal cells exhibit increases in inhibitory postsynaptic potentials. However, only $20 \%$ of the cells survive. Baraban et al. [12] then showed that grafted MGE cells exhibit anti-epileptic effects in a Kv1.1 mutant mouse model of generalized epilepsy and demonstrated synaptogenesis at posttransplantation day 30 by electron microscopy, in addition to increases in inhibitory tone. Zipancic et al. [13] demonstrated reduced seizure threshold after transplantation of MGE cells into the adult hippocampus of a rodent model receiving a neurotoxin against GABAergic subpopulations in the hippocampus. However, only $20 \%$ of the grafted cells survived. None of these previous studies included paired cell recordings to convincingly demonstrate that the surviving MGE-derived interneurons were directly making functional inhibitory synapses on the 
surrounding pyramidal cells as the etiology of the antiepileptogenic effect.

Assessment of cell survival indicates that only $20 \%$ of transplanted cells survive $[12,13,18]$. In addition, although migration occurs up to $5 \mathrm{~mm}$ from the site of transplantation into early postnatal host neocortex, cell density is highest at the site of transplant and then falls off dramatically after $\sim 1 \mathrm{~mm}$ [18]. In adult hosts, transplants migrate only 1 to $2 \mathrm{~mm}$ with similar drop-off $[10,13,18]$. These data raise the question of how such limited numbers of surviving focally transplanted cells can successfully impact a network-based widespread disease such as epilepsy. Although patched adjacent pyramidal cells exhibit increases in inhibitory postsynaptic potentials (IPSPs), the lack of paired-cell recordings raises the possibility that the increase in GABAergic inhibition may not be arising from synaptic activity of the transplanted cells themselves [13, 18]. If the anti-epileptic effect of transplanted MGE cells is based on increased inhibitory tone arising from the synaptic connections of the transplanted cells, one would expect a relationship between the number of surviving cells and their anti-epileptic effect. However, this relationship has not been previously investigated. In addition, although transplants into the neonatal cortex have shown anti-epileptogenesis [12], indicating a potential therapy for newborns destined to develop seizures, it is unclear if similar transplants into the adult neocortex will be an effective anti-epileptic therapy for ongoing seizures. Although medication is generally the first-line therapy for epilepsy, anticonvulsant drugs are not always effective in all patients with epilepsy. Surgical removal of the epileptic focus provides an alternative strategy for treating focal epilepsy, but may cause significant side effects and can be morbid to adjacent functional cortex. MGE cell therapy for focal neocortcal epilepsy is potentially equally effective as surgical therapy with less morbidity.

In this study, we demonstrate the ability of MGE cells to cause ictal attenuation in a model of focal neocortical ictal epileptiform events in adult rodent. This effect was pronounced, occurring as early as 2.5 weeks after cell transplantation. Although transplanted MGE cells demonstrate neurite outgrowth as early as 1 week after transplantation in adults (Fig. 1e), it is unlikely that such a small number of immature cells would have such a profound impact on ictal epileptiform events propagation using a synaptic mechanism. Likewise, variations in cell survival should dramatically influence a synaptic effect. Although synaptic mechanisms may play a partial role in discharge attenuation, other mechanisms are likely involved. One possibility is tonic inhibition from extrasynaptic GABA onto the $\mathrm{GABA}_{\mathrm{A}}-\mathrm{R} \alpha 4$ subunit [41]. GABA-mediated tonic inhibition has been shown to inhibit seizure activity, such as the anticonvulsant vigabatrin, which inhibits GABAtransaminase, increases the concentration of GABA in the brain [42], resulting, in part, from nonvesicular release of GABA [43]. Indeed, Baraban et al. [12] demonstrated an increase in extrasynaptic GABA in a previous study of MGE transplants. However, increasing synaptic and extrasynaptic GABA pharmacologically was not efficient in attenuating 4-AP events in a previous study [28]. Likewise, one would expect cell survival to influence extrasynaptic GABA levels as well. A further study of the mechanism of MGE transplants using $\mathrm{GABA}_{\mathrm{A}}-\mathrm{R} \alpha 4$ knockout mice and in vitro paired recordings was clearly warranted.

Another possible mechanism is transplant-induced microglial reactivity. Although little is known about microglial response to embryonic transplant cell in adult brain, we did not find any increase in microglia proliferation around our transplants rendering this mechanism less likely ("see Supplemental Material for details").

Previous studies have shown that transplants of dorsal or ventral regions of E13.5 MGE, placed into the isochronic MGE in utero [16], or into the neonatal neocortex [15], result in a bias in favor of SST+interneurons in the dMGE transplants, and PV+interneurons in the vMGE transplants. In this study, however, we did not see evidence for this bias (Fig. 2). The reason for this discrepancy is not clear, but it may have to do with differential survival of interneuron subgroups after transplantation into the adult cortex $v s$ embryonic or neonatal hosts. In addition, the relatively low percentage of PV colabeling from either source (20\%; Fig. 2), and relatively high percentage number of aspiny, multipolar cells that did not express either marker, may suggest that transplantation into adult mice may result in inadequate excitatory drive to detectably induce this activity-regulated protein.

Consistent with the similar composition of dMGE and vMGE transplants, there was no difference in reduction of ictal power from either transplant source (Fig. 3). Future studies using more spatially and temporally defined regions may better explore the ability of distinct interneuron subgroups to influence ictal discharges in this and other models. In addition, as electrophysiologically and neurochemically-defined cortical interneurons have recently been generated from embryonic stem cells, it may soon be possible to generate specific interneuron types from this resource [44], opening up the possibility of studies that determine which interneuron subclass is most efficacious for blocking different types of discharges. Furthermore, inter-animal variability has important implications in cell therapy [45]. Seizure duration, susceptibility to therapy and response can all be animal-dependent. Hence, response to therapy must be assessed in groups of animals and compared with like controls.

In summary, we provide evidence that MGE progenitor cell transplantation attenuates ictal power in the adult brain as early as 2.5 weeks after transplantation in a manner that is independent of cell survival greater than a low threshold. These findings suggest that the precise mechanism of this 
cell-based therapy is not purely synaptic. The reproducibility and ease of induction of ictal epileptiform events make the 4-AP model a useful rapid-throughput screen for the efficacy of cell-based therapy for focal neocortical seizures.

Acknowledgments This work was supported by the National Institutes of Health/National Institute of Neurological Disorders and Stroke RO1 NS49482 (T.H.S) and R21 NS054866 (S.A.A.), and The C.V. Starr Foundation (E.D. and S.A.A). Full conflict of interest disclosure is available in the electronic supplementary material for this article.

\section{References}

1. Spencer S, Huh L. Outcomes of epilepsy surgery in adults and children. Lancet Neurol 2008;7:525-537.

2. Engel JJ, Van Ness PC, Rasmussen TB, Ojemann LM. In: Surgical treatment of the epilepsies. Engel JJ, ed. New York: Raven Press, 1993:609-623.

3. DeFelipe J. Chandelier cells and epilepsy. Brain 1999;122:1807-1822.

4. Baraban SC. Neuropeptide Y and limbic seizures. Rev Neurosci 1998;9:117-128.

5. Lindvall O, Bjorklund A. Intracerebral grafting of inhibitory neurons. A new strategy for seizure suppression in the central nervous system. Adv Neurol 1992;57:561-569.

6. Thompson KW. Genetically engineered cells with regulatable GABA production can affect afterdischarges and behavioral seizures after transplantation into the dentate gyrus. Neuroscience 2005;133:1029-1037.

7. Guttinger M, Fedele D, Koch P, et al. Suppression of kindled seizures by paracrine adenosine release from stem cell-derived brain implants. Epilepsia 2005;46:1162-1169.

8. Li T, Steinbeck JA, Lusardi T, et al. Suppression of kindling epileptogenesis by adenosine releasing stem cell-derived brain implants. Brain 2007;130:1276-1288.

9. Anderson SA, Eisenstat DD, Shi L, Rubenstein JL. Interneuron migration from basal forebrain to neocortex: dependence on Dlx genes. Science 1997;278:474-476.

10. Wichterle H, Garcia-Verdugo JM, Herrera DG, Alvarez-Buylla A. Young neurons from medial ganglionic eminence disperse in adult and embryonic brain. Nat Neurosci 1999;2:461-466.

11. Waldau B, Hattiangady B, Kuruba R, Shetty AK. Medial ganglionic eminence-derived neural stem cell grafts ease spontaneous seizures and restore GDNF expression in a rat model of chronic temporal lobe epilepsy. Stem Cells 2010;28:1153-1164.

12. Baraban SC, Southwell DG, Estrada RC, et al. Reduction of seizures by transplantation of cortical GABAergic interneuron precursors into Kv1.1 mutant mice. Proc Natl Acad Sci U S A 2009;106:15472-15477.

13. Zipancic I, Calcagnotto ME, Piquer-Gil M, Mello LE, lvarezDolado M. Transplant of GABAergic precursors restores hippocampal inhibitory function in a mouse model of seizure susceptibility. Cell Transplantation 2010;19:549-564.

14. Wonders CP, Anderson SA. The origin and specification of cortical interneurons. Nat Rev Neurosci 2006;7:687-696.

15. Wonders CP, Taylor L, Welagen J, et al. A spatial bias for the origins of interneuron subgroups within the medial ganglionic eminence. Dev Biol 2008;314:127-136.

16. Flames N, Pla R, Gelman DM, et al. Delineation of multiple subpallial progenitor domains by the combinatorial expression of transcriptional codes. J Neurosci 2007;27:9682-9695.

17. Fogarty M, Grist M, Gelman D, et al. Spatial genetic patterning of the embryonic neuroepithelium generates GABAergic interneuron diversity in the adult cortex. J. Neurosci 2007;27:10935-10946.
18. Alvarez-Dolado M, Calcagnotto ME, Karkar KM, et al. Cortical inhibition modified by embryonic neural precursors grafted into the postnatal brain. J Neurosci 2006;26:7380-7389.

19. Southwell DG, Froemke RC, Alvarez-Buylla A, Stryker MP, Gandhi SP. Cortical plasticity induced by inhibitory neuron transplantation. Science 2010;327:1145-1148.

20. Ulbricht W, Wagner HH. Block of potassium channels of the nodal membrane by 4-aminopyridine and its partial removal on depolarization. Pflugers Arch 1976;367:77-87.

21. Benardo LS. Recruitment of GABAergic inhibition and synchronization of inhibitory interneurons in rat neocortex. J Neurophysiol 1997;77:3134-3144.

22. Zhao M, Ma H, Suh M, Schwartz TH. Spatiotemporal dynamics of perfusion and oximetry during ictal discharges in the rat neocortex. J Neurosci 2009;29:2814-2823.

23. Brückner C, Heinemann U. Effects of standard anticonvulsant drugs on different patterns of epileptiform discharges induced by 4-aminopyridine in combined entorhinal cortex-hippocampal slices. Brain Research 2000;859:15-20.

24. Brückner C, Stenkamp K, Meierkord H, Heinemann U. Epileptiform discharges induced by combined application of bicuculline and 4-aminopyridine are resistant to standard anticonvulsants in slices of rats. Neurosci Lett 1999;268:163-165.

25. Motamedi GK, Salazar P, Smith EL, et al. Termination of epileptiform activity by cooling in rat hippocampal slice epilepsy models. Epilepsy Res 2006;70:200-210.

26. Schuchmann S, Buchheim K, Meierkord H, Heinemann U. A relative energy failure is associated with low-Mg2 + but not with 4- aminopyridine induced seizure-like events in entorhinal cortex. J Neurophysiol 1999;81:399-403.

27. Wahab A, Albus K, Gabriel S, Heinemann U. In search of models of pharmacoresistant epilepsy. Epilepsia 2010;51:154-159.

28. Yamaguchi S, Rogawski MA. Effects of anticonvulsant drugs on 4aminopyridine-induced seizures in mice. Epilepsy Res 1992;11:9-16.

29. De Curtis M, Gnatkovsky V. Reevaluating the mechanisms of focal ictogenesis: The role of low-voltage fast activity. Epilepsia 2009;50:2514-2525.

30. Xu Q, Cobos I, De La Cruz E, Rubenstein JL, Anderson SA. Origins of cortical interneuron subtypes. J Neurosci 2004;24:2612-2622.

31. Buzsaki G, Bayardo F, Miles R, Wong RK, Gage FH. The grafted hippocampus: an epileptic focus. Exp Neurol 1989;105:10-22.

32. Buzsaki G, Masliah E, Chen LS, et al. Hippocampal grafts into the intact brain induce epileptic patterns. Brain Res 1991;554:30-37.

33. Thompson WG. Successful brain grafting. NY Med J 1890;51:701702 .

34. Noble M. Can neural stem cells be used as therapeutic vehicles in the treatment of brain tumors? Nat Med 2000;6:369-370.

35. Ebert AD, Barber AE, Heins BM, Svendsen CN. Ex vivo delivery of GDNF maintains motor function and prevents neuronal loss in a transgenic mouse model of Huntington's disease. Exp Neurol 2010;224:155-162.

36. Emborg ME, Ebert AD, Moirano J, et al. GDNF-secreting human neural progenitor cells increase tyrosine hydroxylase and VMAT2 expression in MPTP-treated cynomolgus monkeys. Cell Transplant 2008; 17:383-395.

37. Ebert AD, Beres AJ, Barber AE, Svendsen CN. Human neural progenitor cells over-expressing IGF-1 protect dopamine neurons and restore function in a rat model of Parkinson's disease. Exp Neurol 2008;209:213-223.

38. Suzuki M, McHugh J, Tork C, et al. GDNF secreting human neural progenitor cells protect dying motor neurons, but not their projection to muscle, in a rat model of familial ALS. PLoS One 2007;2:e689.

39. Thompson KW, Suchomelova LM. Transplants of cells engineered to produce GABA suppress spontaneous seizures. Epilepsia 2004;45:4-12. 
40. Shetty AK, Zaman V, Hattiangady B. Repair of the injured adult hippocampus through graft-mediated modulation of the plasticity of the dentate gyrus in a rat model of temporal lobe epilepsy. J Neurosci 2005;25:8391-8401.

41. Belelli D, Harrison NL, Maguire J, et al. Extrasynaptic GABA receptors: form, pharmacology, and function. J Neurosci 2009;29:12757-12763.

42. Petroff OA, Behar KL, Mattson RH, Rothman DL. Human brain g-aminobutyric acid levels and seizure control following initiation of vigabatrin therapy. J Neurochem 1996;67:2399-2404.
43. Wu Y, Wang W, Richerson GB. Vigabatrin induces tonic inhibition via GABA transporter reversal without increasing vesicular GABA release. J Neurophysiol 2003;89:2021-2034.

44. Maroof AM, Brown K, Shi S-H, Studer L, Anderson SA. Prospective isolation of cortical interneuron precursors from mouse embryonic stem cells. J Neurosci 2010;30:4667-4675.

45. Zbinden S, Clavijo LC, Kantor B, et al. Interanimal variability in preexisting collaterals is a major factor determining outcome in experimental angiogenesis trials. Am J Physiol Heart Circ Physiol 2007;292:H1891-H1897. 